\title{
Analytical applications of biomimetic recognition elements
}

\author{
María Cruz Moreno-Bondi ${ }^{1} \cdot$ Elena Benito-Peña ${ }^{1}$
}

Published online: 23 December 2015

(C) Springer-Verlag Berlin Heidelberg 2015

With the advent of the 21 st century, public awareness on issues related to human and animal health, food safety, or environmental pollution has increased considerably and a great deal of research and substantial investments have focused on the development of novel tools for clinical diagnostics, food control, or continuous in situ environmental monitoring [1].

Molecular recognition refers to the process by which a specific complex is formed between two molecules; the host is, typically, a macromolecule or molecular assembly, whereas the guest is the target molecule that binds to the former, typically the analyte in this context [2]. At the beginning, this process was primarily based on the use of natural elements such as enzymes, antibodies, nucleic acids, or biological receptors isolated from living systems. Biomolecular receptors show high ligand specificity and affinity, as a result of millions of years of evolution. However, their analytical application is sometimes hampered, among other problems, by their relative instability in non-physiological conditions, the low production yields, or their high cost, together with the social concern raised by the use of laboratory animals.

The term "biomimetic" was coined by the polymath Otto Schmitt in 1957 to designate biologically inspired design, adaptation, or derivation from Nature. This word is derived from the

Published in the topical collection Analytical Applications of Biomimetic Recognition Elements with guest editors Maria C. Moreno-Bondi and Elena Benito-Peña.

María Cruz Moreno-Bondi

mcmbondi@ucm.es

Elena Benito-Peña

elenabp@ucm.es

1 Department of Analytical Chemistry, Faculty of Chemistry, Universidad Complutense de Madrid, 28040 Madrid, Spain
Greek word biomimesis, which adjoins bios (life), and mimesis (to imitate) [3]. The development of new molecular recognition receptors, not occurring naturally, that can be engineered and prepared by human beings in the laboratory but mimic the behavior of natural molecules, emerged as an attractive alternative to overcome some of the limitations of their biological counterparts. Synthetic receptors have been defined recently as "synthetic, semisynthetic, or rationally designed biological molecules created with the purpose of selectively binding a single compound or group of structurally similar compounds" [4]. In principle, these artificial materials can be custom designed for a wide range of applications and are characterized by excellent physicochemical stability, durability, ease of storage, and affordability compared with those of biological receptors.

Therefore, the ability to develop tailored recognition elements, with a broad structural and chemical diversity, can be expected to lead to an entirely new plethora of applications, not only for biosensing or analytical separations but also for drug delivery, sustained/controlled release of active principles, or environmental remediation, among others. The main groups of synthetic bespoke receptors currently in use include genetically engineered proteins and cells, synthetic peptides, molecularly imprinted polymers (MIPs), supramolecular receptors, oligonucleotides [locked nucleic acids (LNAs), peptide nucleic acids (PNAs)], and aptamers [2, 4]. Moreover, different methodologies, including computational chemistry, combinatorial chemistry, or phage display, have been employed to discover or design new recognition elements for specific targets [2].

The development of biosensors based on nanomaterials and nanodevices has emerged as an exciting area under fast development $[5,6]$. New artificial materials engineered to the nanoscale (magnetic, metallic, and silica nanoparticles; noble metal nanoparticles; quantum dots, etc.) can be conjugated with molecular recognition elements, allowing a significant improvement in the interactions with the analyte and generating stronger 
signal responses. Moreover, the local field effects of radiation on nanomaterials are several orders of magnitude higher than the corresponding bulk effects so that the application of optically active metallic and quantum dot nanomaterials has boosted the development of newer techniques of interest in sensing and bioimaging, such as surface-enhanced Raman scattering (SERS), local surface plasmon resonance (LSPR), metalenhanced fluorescence (MEF), to name a few. Size reduction to the nanoscale also enables an improvement in the detection speed, a decrease of the sample volume, cost-saving benefits, and an increase in the degree of multiplexing, opening the door to the production of sensing platforms that combine a variety of bio- and biomimetic molecular recognition elements for general diagnostic and detection purposes. However, although nanotechnology-based methods are routinely applied today in the research labs, their use with commercial purposes for clinical diagnosis and medical treatments is still in its infancy [6].

This topical collection of articles gives an insight into the recent advances and future prospects of biomimetic recognition elements in the analytical chemistry field. Research and development in this area has rapidly expanded in the last two decades and the contributors to this topical collection have reviewed the application of peptides, carbohydrates, engineered bacteriophages, and molecularly imprinted polymers to the development of sensors, diagnostics assays, and analytical separations. We would like to thank all the authors for submitting excellent contributions for this topical issue. Thanks to the referees for their careful reviews and constructive criticisms and our deep gratitude to the editorial team of Analytical and Bioanalytical Chemistry for their highly professional and valuable cooperation.

\section{References}

1. Li S, Jiang W, Wang ZM, Jiang Y (eds) (2013) Nanoscale sensors in lecture notes in nanoscale science and technology. Springer, BerlinHeidelberg

2. Zourob M (ed) (2007) Recognition receptors in sensors. Springer, Berlin-Heidelberg

3. Bhushan B (ed) (2010) Springer handbook of nanotechnology. Springer, Berlin-Heidelberg

4. Piletsky SA, Whitcombe MJ (eds) (2013) Designing receptors for the next generation of biosensors. Springer, Berlin-Heidelberg

5. Kelley SO, Mirkin CA, Walt DR, Ismagilov RF, Toner M, Sargent EH (2014) Advancing the speed, sensitivity, and accuracy of biomolecular detection using multi-length-scale engineering. Nat Nanotechnol 9:969-80

6. Lu J, Wu N (2014) Biosensors based on nanomaterials and nanodevices. CRC Press, Boca Raton

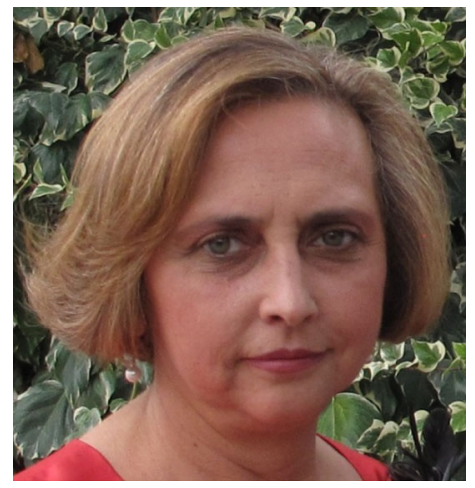

Maria C. Moreno-Bondi received her Ph.D. in Analytical Chemistry at Complutense University (UCM) of Madrid (Spain) in 1990. In 2008 she was promoted to full professor at the Department of Analytical Chemistry of UCM. She has carried out several research stages as a research fellow and as visiting professor at Columbia University (New York, 1988/89 and 2013), the Naval Research Lab (Washington DC, 2015), the Oak

Ridge National Lab (Tennessee, 1997), and the Karl-Franzens University (Austria, 1989), working on the characterization of dendrimers as well as on the development of fluorescent based optical fiber sensors, biosensors, and microarrays. She has received the Young Researcher's Award from the Spanish Society of Analytical Chemistry in 1993 and the Research Award in Analytical Chemistry from the Royal Spanish Society of Chemistry in 2010. Her research interests lie in the development of optical chemical sensors and biosensors and their applications to environmental and food analysis as well as in the development of molecularly imprinted polymers for sensing and separation purposes. She is currently Department Chair of the Analytical Chemistry Department at UCM, President of the Society of Applied Spectroscopy, and co-leader of the Chemical Optosensors and Applied Photochemistry Group (GSOLFA). She serves as a member of the International Advisory Board of the journal Analytical and Bioanalytical Chemistry and in the Permanent Steering Committee of ASCOS (“Advanced Study Courses on Optical Sensors”).

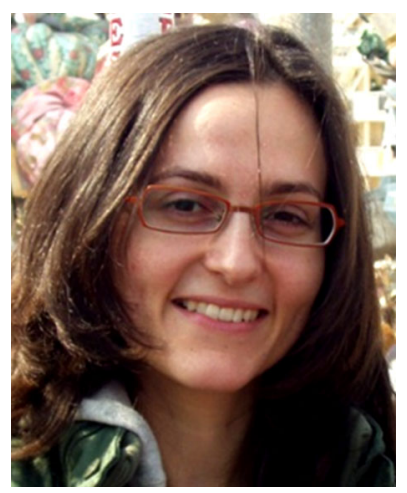

Elena Benito-Peña received her $\mathrm{Ph} . \mathrm{D}$. in Chemistry from Complutense University (Madrid) in 2006. In 2005 she moved to the private sector, working at the Chromatography Lab of Interlab Group (now Labaqua). In 2006, she returned to Complutense University, where she is currently a Postdoctoral Research Associate in the GSOLFA group at the Department of Analytical Chemistry. In 2009 she was awarded a Postdoctoral fellowship from the Spanish Government (MECFECYT) to work with Professor David Walt at Tufts University (20092011) in the development of multiplexed optical fiber bundle-based biosensor microarrays and InfoBiology. Her research interests are optical sensors and biosensors, microarray biosensing platforms, synthesis of biomimetic elements for sensor and separation purposes, and their application to food, clinical, and environmental analysis. 